\title{
It is time for TEG in pediatric trauma: unveiling meaningful alterations in children who undergo massive transfusion
}

\author{
Ryan Phillips ${ }^{1,2} \cdot$ Hunter Moore $^{2,3} \cdot$ Denis Bensard ${ }^{1,2,3} \cdot$ Niti Shahi $^{1,2} \cdot$ Gabrielle Shirek $^{1,2} \cdot$ Marina L. Reppucci ${ }^{1,2}$. \\ Maxene Meier ${ }^{4}$ John Recicar ${ }^{1}$. Shannon Acker ${ }^{1,2}$. John Kim ${ }^{5}$. Steven Moulton ${ }^{1,2}$
}

Accepted: 1 June 2021 / Published online: 17 September 2021

○ The Author(s), under exclusive licence to Springer-Verlag GmbH Germany, part of Springer Nature 2021

\begin{abstract}
Background Hemorrhage is the leading cause of preventable death in pediatric trauma patients. In adults, goal-directed thrombelastography (TEG) has been shown to reduce mortality when used to guide massive transfusion (MT) resuscitation. There remains a paucity of data on the utility of TEG in directing resuscitation of pediatric trauma patients. We hypothesize that abnormalities on admission TEG will differ in pediatric trauma patients who undergo MT, compared to those who do not. Methods Pediatric patients ( $\leq 18$ years) who were highest level trauma activations at two trauma centers from 2015 to 2018 were analyzed. We included patients who had admission TEGs and excluded those who did not. Patients were stratified into two groups: those who received MT ( $>40 \mathrm{cc} / \mathrm{kg}$ total blood product within $6 \mathrm{~h}$ of admission) and those who did not. We defined TEG abnormalities based on each institution's normative values and compared TEG abnormalities between the groups.

Results Of 117 children included, 39 had MT. MT patients had higher injury severity scores (30 vs. 23, $p=0.0004$ ), lactates levels (7.0 vs. $3.5, p<0.001)$, base deficit levels ( -12.2 vs. $-5.8, p<0.001)$, and INR values $(1.8$ vs. $1.3, p<0.001)$. MT patients had significantly shortened alpha-angles $(35.9 \%$ vs. $15.4 \%, p=0.023)$, maximum amplitude (MA) values $(43.6 \%$ vs. $10.3 \%, p<0.001$ ), and significantly lower platelet counts (165 vs. $281, p<0.001)$ compared to those who did not receive MT. There was no difference in the trends in R-time, LY30 (lysis or shutdown), or fibrinogen concentration between the groups. Logistic regression identified a decreased MA as a significant predictor for MT [OR 3.68 (CI 1.29-10.52)]

Conclusions Pediatric trauma patients who undergo MT are more likely to have lower alpha-angles and MA values, as well as lower platelet counts. These findings support the use of TEG to identify hemorrhaging pediatric trauma patients, who may benefit from cryoprecipitate and/or platelet transfusions. TEG provides real-time information on coagulation status, which may expedite the delivery of specific blood products during trauma resuscitation.
\end{abstract}

Level of evidence: Level III Type of study: Retrospective comparative study.

Keywords Trauma-induced coagulopathy $\cdot$ Pediatric trauma $\cdot$ Thrombelastography $\cdot$ TEG $\cdot$ Goal-directed hemostatic resuscitation

John Kim and Steven Moulton denotes co-senior authors.

Ryan Phillips

Ryan.Phillips@childrenscolorado.org

1 Division of Pediatric Surgery, Children's Hospital Colorado, Aurora, CO, USA

2 Department of Surgery, University of Colorado School of Medicine, Aurora, CO, USA

3 Department of Surgery, Denver Health Medical Center, Denver, CO, USA
4 The Center for Research in Outcomes for Children's Surgery, Center for Children's Surgery, University of Colorado School of Medicine, Aurora, CO, USA

5 Department of Pediatrics, School of Medicine, Heart Institute, Children's Hospital Colorado, University of Colorado, Aurora, CO, USA 


\section{Introduction}

Trauma accounts for one million pediatric deaths globally each year, with over eighty percent of all preventable deaths associated with hemorrhage [1]. Pediatric trauma patients who require massive transfusion (MT) have a mortality rate of over $40 \%$, yet few studies aim to optimize care for these children [2, 3]. Following publication of adult data, there has been a gradual shift led by pediatric trauma centers in the U.S., to restrict crystalloid administration and deliver early balanced blood product resuscitation for children with evidence of hemorrhagic shock [4]. Recent data from a multicenter civilian retrospective analysis on pediatric patients-receiving transfusion volumes of $\geq 20 \mathrm{~mL} / \mathrm{kg}$ of $\mathrm{pRBC}$ found a survival benefit with more balanced blood product ratios [5].

It is important to recognize ongoing hemorrhage due to trauma-induced coagulopathy, because up to $60 \%$ of children have evidence of trauma-induced coagulopathy on admission [6-8]. Traditionally, the degree of coagulopathy is quantified by conventional coagulation tests (CCTs), such as prothrombin time (PT), international normalized ratio (INR), partial thromboplastin time (PTT). Unfortunately, CCTs inadequately characterize the extent of coagulopathy in severe trauma [9]. On the other hand, there is a randomized control trial and emerging military evidence in the adult literature that goal-directed transfusions based on viscoelastic assays of hemostasis, such as thrombelastography (TEG) not only improves survival but reduces waste of blood product components $[10,11]$. The evidence for the use of viscoelastic assays [TEG or rotational thromboelastometry (ROTEM)] is strong enough that the American College of Surgeons (ACS) Trauma Quality Improvement Program now recommends the use of viscoelastic assays, if available, to guide resuscitation for adult patients at risk for massive hemorrhage following trauma [12].

TEG is a viscoelastic point-of-care test of whole blood global hemostasis that measures the rapidity and strength of blood clot formation followed by its degradation by fibrinolysis [7, 8]. TEG provides data from initiation of clot formation via platelet-fibrin interaction (demonstrated by the R-time), through platelet aggregation and clot strengthening (demonstrated by the alpha-angle), to the maximal clot strength (maximal amplitude) [9]. The clinical treatments of coagulopathy demonstrated by abnormalities in these values is generally accepted as: a prolonged R-time is treated with fresh frozen plasma (FFP), a decreased alpha-angle is treated with cryoprecipitate, and a low MA is treated with platelet transfusion.

Although goal-directed TEG has been shown to reduce mortality in adult trauma patients [13], there remains a paucity of data on TEG's utility in directing resuscitation of pediatric trauma patients. We hypothesize that abnormalities on admission TEG will differ in pediatric trauma patients who undergo MT, compared to those who do not. Such differences may have clinical implications for guiding activation of MT among the pediatric trauma population.

\section{Methods}

\section{Study setting}

The local Institutional Review Board approved this study. The two study centers involved are an ACS verified Level I pediatric trauma center (PTC), and an ACS verified Level II PTC. The study was conducted from January 2015 to December 2018. Pediatric patients ( $\leq 18$-years old) who met the highest-level trauma activation criteria and had a TEG within two hours of arrival were included. Exclusion criteria included blood product transfusion before arrival, lack of coagulation studies within two hours after arrival, and burn injuries.

\section{Data collection}

Two prospectively maintained trauma registries were used to identify all patients $\leq 18$-years old, who had an admission TEG or r-TEG. The following demographic data points were extracted from the registries: age, weight, gender, mechanism of injury (blunt vs. penetrating), Glasgow Coma Scale (GCS), and Injury Severity Score (ISS). Emergency department (ED) vital signs and admission laboratory values were also collected. All included injured patients had CCTs [defined as PT, INR, PTT, platelet count, D-dimer, and fibrinogen] and other laboratory values [lactate and base deficit (BD)].

The primary aim of the study was to identify and compare TEG abnormalities between those who had activation of a MT versus those who did not (non-MT group). MT was defined as $40 \mathrm{cc} / \mathrm{kg}$ of total blood products within the first six hours of arrival to either PTC or its activation was at the discretion of the physician at the time of trauma based on predicted volume loss and other clinical parameters independent of TEG results. [2]. Secondary aims were to compare the total number of ventilator days, intensive care unit (ICU) length of stay (LOS), hospital LOS, and mortality.

\section{TEG processing}

The ACS verified Level I PTC utilizes kaolin TEG (k-TEG). Whole blood specimens are obtained during the primary or secondary survey at the request of the trauma team leader. 
The clinical laboratory at this institution utilizes a TEG 5000 analyzer (Haemonetics Corp, Braintree, Massachusetts). The k-TEG parameters collected for each subject included the R-time, alpha-angle, maximum amplitude (MA), and percent clot lysis $30 \mathrm{~min}$ after MA is achieved (LY30). Institutional normative values for the k-TEG are R-time of 4-9 min, alpha-angle of 59-74 degrees, MA of 55-74 mm, and LY30 of $0.9-3 \%$. The significance of each parameter has been described previously [14].

The ACS verified Level II PTC utilizes rapid TEG (r-TEG). This site also uses a TEG-5000 Analyzer (Haemonetics Corp, Braintree, Massachusetts). Whole blood is collected in vacuum tubes with citrate added to prevent clotting before analysis. This assay adds tissue factor plus kaolin reagents to the whole blood sample immediately before test initiation to expedite the results. R-TEG yields the following variables: activated clotting time (ACT), R-value, alpha-angle, MA, and LY30. The significance of each r-TEG parameter has also been previously described $[6,8,9]$.

At both PTC, TEG is utilized over ROTEM given its availability. TEG values are available quickly. Results for $\mathrm{k}$-TEG can be viewed in real-time electronically in the trauma bay, but full results take approximately $45 \mathrm{~min}$. Results for r-TEG, such as ACT, K-time and R-time are available within minutes. Full results can take as quick as 15 min depending on how quickly a patient is clotting. However, neither institutions have guidelines in place for the activation of MT based on TEG values.

\section{Statistical analysis}

Patients were stratified into two groups for comparison: those who had activation of MT and those who did not (nonMT group). We defined TEG abnormalities based on each institution's normative values and compared TEG abnormalities between the groups. Data are reported as frequencies and percentages, means and standard deviations, or medians and interquartile range (IQR) depending on their distribution. Univariate testing was performed with Student's t tests.

Correlation of k-TEG and r-TEG values with relevant coagulation tests were assessed using Pearson's correlation coefficients. ACT, R-time, and alpha-angle were correlated with PT, INR, and PTT. Next, alpha-angle and MA were correlated with fibrinogen concentration and platelet counts using Pearson's correlation coefficients. A logistic regression model was performed to determine if any significantly abnormal k-TEG or r-TEG values were predictors of the need for MT. Alpha-angle and MA were dichotomized as abnormal and normal based on their respective institutional normative values. The variables included in the final logistic regression analyses were age, GCS, alpha-angle, and MA. Significance was set at $p<0.05$ for all analyses. $\mathrm{R}$ version 3.6.1 software (R Foundation for Statistical Computing,
Vienna, Austria, http://www.R-project.org/) was utilized for data cleaning, analysis, and visualization.

\section{Results}

\section{Patient admission characteristics}

A total of 117 patients met highest level of trauma activation criteria and had a k-TEG or r-TEG result within the first two hours of presentation to either PTC. Thirtynine patients had MT. In the non-MT group, 40\% (31/78) received at least one blood product transfusion. The baseline demographics and injury characteristics are shown in Table 1. Patients who received MT were significantly younger (12.4 vs. 14.9-years old, $p=0.016$ ) and weighed less (50 vs. $63 \mathrm{~kg}, p=0.013)$. We did not find a difference

Table 1 Demographic, injury, and outcome data by massive transfusion status

\begin{tabular}{|c|c|c|c|}
\hline & $\begin{array}{l}\text { Massive } \\
\text { transfusion } \\
(n=39)\end{array}$ & $\begin{array}{l}\text { No massive } \\
\text { transfusion } \\
(n=78)\end{array}$ & $p$-value \\
\hline Weight (kg) & $50.1(28.8)$ & $63.7(26.4)$ & 0.013 \\
\hline Age, years & $12.4(7.3)$ & $14.9(3.9)$ & 0.016 \\
\hline Male, $n(\%)$ & $31(79.5 \%)$ & $61(78.2 \%)$ & 1.000 \\
\hline Blunt mechanism, $n(\%)$ & $21(53.8 \%)$ & $43(55.1 \%)$ & 1.000 \\
\hline ISS & $30.0(13.3)$ & $20.0(16.6)$ & 0.001 \\
\hline SIPA & $1.4(0.7)$ & $0.95(0.3)$ & 0.001 \\
\hline GCS & $8.7(5.7)$ & $11.3(5.2)$ & 0.014 \\
\hline \multicolumn{4}{|c|}{ Admission laboratory values } \\
\hline INR & $1.8(0.8)$ & $1.3(0.32)$ & $<0.001$ \\
\hline PT & $20.8(6.0)$ & $16.1(2.8)$ & $<0.001$ \\
\hline Abnormal, $n(\%)$ & $36(94.7 \%)$ & $42(54.5 \%)$ & $<0.001$ \\
\hline PTT & $59.9(49.7)$ & $29.8(8.7)$ & $<0.001$ \\
\hline Abnormal, $n(\%)$ & $20(51.3 \%)$ & $18(23.4 \%)$ & 0.002 \\
\hline Lactate & $7.0(4.9)$ & $3.5(2.9)$ & $<0.001$ \\
\hline Base deficit & $-12.2(6.94)$ & $-5.8(4.1)$ & $<0.001$ \\
\hline Platelet count & $165.6(107.22)$ & $281.9(107.36)$ & $<0.001$ \\
\hline Fibrinogen & $170.0(152.7)$ & $227.5(80.9)$ & 0.203 \\
\hline \multicolumn{4}{|l|}{ Clinical outcomes } \\
\hline Ventilator, days & $5.9(7.4)$ & $2.5(5.1)$ & 0.004 \\
\hline $\begin{array}{l}\text { Intensive care unit stay, } \\
\text { days }\end{array}$ & $10.3(11.6)$ & $4.1(6.7)$ & $<0.001$ \\
\hline $\begin{array}{l}\text { Hospital length of stay, } \\
\text { days }\end{array}$ & $16.9(15.6)$ & $8.8(11.2)$ & 0.001 \\
\hline Mortality, n (\%) & $7(17.9 \%)$ & 7 (9.0\%) & 0.268 \\
\hline
\end{tabular}

Bold values indicate statistical significance

kg kilograms, ISS injury severity score, SIPA age-adjusted shock index, GCS Glasgow coma score, INR international normalized ratio

Continuous variables presented as mean (standard deviation), Categorical values presented as frequency (percentage) 
in gender (79\% vs. $78 \%$ male) or mechanism of injury (54\% vs. $55 \%$ blunt injury). Pediatric trauma patients who underwent MT had higher ISS (30 vs. 20, $p=0.001$ ) and a lower GCS (8.7 vs. $11.3, p=0.014)$.

Admission INR values ( 1.8 vs. $1.3, p<0.001)$ and lactate levels (7.0 vs. 3.5, $p<0.001)$ were significantly higher in patients who received MT. In addition, BD levels $(-12.2$ vs. $-5.8, p<0.001)$ values were significantly greater in nonMT patients. Those who received MT had significantly lower platelet counts ( 165 vs. $281, p<0.001$ ) on admission, but no statistical difference was seen in their admission fibrinogen concentration ( 227 vs. $170, p=0.203$ ). A breakdown of the clinical outcomes is shown in Table 1 . The overall mortality rate was $12 \%$, with no significant difference between the MT and non-MT cohorts $(17.9 \%$ vs. $9.0 \%, p=0.268)$.

\section{TEG abnormalities in those who received MT $(n=39)$}

Out of a total of 39 patients who underwent MT, 23 (59\%) were treated at hospital I (k-TEG) and $16(41 \%)$ were treated at hospital II (r-TEG). In the MT cohort, $48 \%$ $(11 / 23)$ of patients had a k-TEG alpha-angle $<59$ and $19 \%$ had a r-TEG alpha-angle $<65$. There were $43 \%(10 / 23)$ with a k-TEG MA $<55$ and $43 \%(7 / 16)$ with a r-TEG $<55$. A summary of the percentage of abnormal TEG values for each center is shown in Table 2. Moreover, a breakdown of the median arrival k-TEG and r-TEG values for the MT cohort are shown in Table 3.
TEG abnormalities in those in the non-MT cohort $(n=78)$

Out of a total of 78 patients in the non-MT cohort, 19 (24\%) were treated at hospital I (k-TEG) and remainder were treated at hospital II (r-TEG). Table 3 has a breakdown of this cohort's median arrival k-TEG and r-TEG values. A summary of the percentage of abnormal TEG values for each center is shown in Table 2 and the abnormal values for this cohort are shown in Table 4.

\section{Correlation between TEG/r-TEG and CCTs}

We found a weak correlation between ACT with PT $(r=0.04$, $p=0.720)$, INR $(r=0.03, p=0.810)$, and PTT $(r=0.06$, $p=0.589)$. There was a moderate correlation between R-time with PT $(r=0.33, p<0.001)$, INR $(r=0.36, p<0.001)$, and PTT $(r=0.48, p<0.001)$. Similar to R-time and CCTs, we also found a moderate negative correlation between alpha-angle with PT $(r=-0.45, p<0.001 \mathrm{INR}(r=-0.45$, $p<0.001)$, and PTT $(r=-0.45, p<0.001)$. In addition, there was a weak correlation between the platelet count with alphaangle $(r=0.25, p=0.007)$ and MA $(r=0.19, p=0.042)$.

\section{Combined percentage of TEG abnormalities between those who received MT vs non-MT}

All TEG values were dichotomized as abnormal and normal based on their respective institutional normative values.

Table 2 Abnormal admission TEG parameters in pediatric patients who underwent massive transfusion

\begin{tabular}{|c|c|c|c|c|}
\hline & Normal k-TEG values & k-TEG cohort $(n=23)$ & Normal r-TEG values & r-TEG cohort $(n=16)$ \\
\hline \multicolumn{5}{|l|}{ ACT, s } \\
\hline Elevated & NA & NA & $>191 \mathrm{~s}$ & $12(75.0 \%)$ \\
\hline Decreased & NA & NA & $<74 \mathrm{~s}$ & $0(0.0 \%)$ \\
\hline \multicolumn{5}{|l|}{ R-time } \\
\hline Elevated & $>9 \min$ & $5(21.7 \%)$ & $>0.7$ min & $5(31.3 \%)$ \\
\hline Shortened & $<4$ min & $4(17.4 \%)$ & $<0.3 \mathrm{~min}$ & $1(6.3 \%)$ \\
\hline \multicolumn{5}{|l|}{$\alpha$-angle, degrees } \\
\hline Elevated & $>74^{\circ}$ & $1(4.3 \%)$ & $>79^{\circ}$ & $0(0.0 \%)$ \\
\hline Shortened & $<59^{\circ}$ & $11(36.8 \%)$ & $<65^{\circ}$ & $3(19 \%)$ \\
\hline \multicolumn{5}{|l|}{ MA, mm } \\
\hline Elevated & $>74 \mathrm{~mm}$ & $0(0.0 \%)$ & $>73 \mathrm{~mm}$ & $0(0.0 \%)$ \\
\hline Shortened & $<55 \mathrm{~mm}$ & $10(43.4 \%)$ & $<55 \mathrm{~mm}$ & $7(43.7 \%)$ \\
\hline \multicolumn{5}{|l|}{ LY $30, \%$ of clot lysis } \\
\hline Hyperfibrinolysis & $>\mathbf{3} \%$ & $4(25.0 \%)^{\mathrm{a}}$ & $>\mathbf{3} \%$ & $5(31.2 \%)$ \\
\hline Fibrinolysis shutdown & $<0.9 \%$ & $10(62.5 \%)^{\mathrm{a}}$ & $<0.9 \%$ & $8(50.0 \%)$ \\
\hline
\end{tabular}

Bold values indicate statistical significance

Data presented as $n(\%)$

${ }^{a} 16$ out of the 23 patients had LY30 values available

TEG thrombelastography, ACT activated clotting time, MA maximum amplitude, LY30, \% of clot lysis at $30 \mathrm{~min}$ 
Table 3 Baseline TEG values by massive transfusion status and hospital location

\begin{tabular}{|c|c|c|c|c|c|}
\hline & \multirow[t]{2}{*}{ Normal TEG Values } & \multicolumn{2}{|c|}{ Massive transfusion $(n=39)$} & \multicolumn{2}{|c|}{$\begin{array}{l}\text { No massive transfusion } \\
(n=78)\end{array}$} \\
\hline & & Median & IQR & Median & IQR \\
\hline \multicolumn{6}{|l|}{$\mathrm{ACT}, \mathrm{s}$} \\
\hline Hospital I & NA & NA & NA & NA & NA \\
\hline Hospital II & 74-191 s & 125 & $111-136$ & 121 & $113-128$ \\
\hline \multicolumn{6}{|l|}{ R-time } \\
\hline Hospital I & 4-9 min & 5.2 & $4.1-7.2$ & 4.6 & $3.7-6.5$ \\
\hline $\begin{array}{l}\text { Hospital II } \\
\alpha \text {-angle }\end{array}$ & 0.3-0.7 min & 0.8 & $0.7-0.9$ & 0.8 & $0.7-0.8$ \\
\hline Hospital I & $59-74^{\circ}$ & 62 & $46.7-64.7$ & 62.7 & $54.2-66.5$ \\
\hline Hospital II & $65-79^{\circ}$ & 70.1 & $67.8-73.2$ & 75.3 & $71.5-77.8$ \\
\hline \multicolumn{6}{|l|}{ MA } \\
\hline Hospital I & $55-74 \mathrm{~mm}$ & 55.9 & $42.3-61.4$ & 59.3 & $56.1-63.7$ \\
\hline Hospital II & $55-73 \mathrm{~mm}$ & 55.1 & $50.8-58$ & 60.9 & $57.1-65.7$ \\
\hline \multicolumn{6}{|c|}{ LY30, \% of clot lysis } \\
\hline Hospital I & $0.9-3 \%$ & 0.6 & $0-3.0$ & 0.75 & $0.3-1.5$ \\
\hline Hospital II & $0.9-3 \%$ & 1.05 & $0-4.3$ & 1.6 & $0.4-3.1$ \\
\hline
\end{tabular}

Bold values indicate statistical significance

TEG thrombelastography, NA not applicable, ACT activated clotting time, MA maximum amplitude, LY30, $\%$ of clot lysis at $30 \mathrm{~min}$

Data are presented as median (first and third quartile (IQR))

Table 4 Abnormal admission TEG parameters in pediatric patients who did not undergo massive transfusion

\begin{tabular}{|c|c|c|c|c|}
\hline & $\begin{array}{l}\text { Normal k-TEG } \\
\text { values }\end{array}$ & k-TEG cohort $(n=19)$ & Normal r-TEG values & r-TEG cohort $(n=59)$ \\
\hline \multicolumn{5}{|l|}{$\mathrm{ACT}, \mathrm{s}$} \\
\hline Elevated & NA & NA & $>191 \mathrm{~s}$ & $12(75.0 \%)$ \\
\hline Decreased & NA & NA & $<74 \mathrm{~s}$ & $0(0.0 \%)$ \\
\hline \multicolumn{5}{|l|}{ R-time } \\
\hline Elevated & $>9 \min$ & $4(21.1 \%)$ & $>0.7 \mathrm{~min}$ & $11(18.6 \%)$ \\
\hline Shortened & $<4 \min$ & $7(36.8 \%)$ & $<0.3 \min$ & $1(1.7 \%)$ \\
\hline \multicolumn{5}{|l|}{$\alpha$-angle, degrees } \\
\hline Elevated & $>74^{\circ}$ & $1(5.2 \%)$ & $>79^{\circ}$ & $2(3.4 \%)$ \\
\hline Shortened & $<59^{\circ}$ & $7(36.8 \%)$ & $<65^{\circ}$ & $5(8.4 \%)$ \\
\hline \multicolumn{5}{|l|}{$\mathrm{MA}, \mathrm{mm}$} \\
\hline Elevated & $>74 \mathrm{~mm}$ & $0(0.0 \%)$ & $>73 \mathrm{~mm}$ & $1(1.7 \%)$ \\
\hline Shortened & $<55 \mathrm{~mm}$ & $4(21.1 \%)$ & $<55 \mathrm{~mm}$ & $4(6.7 \%)$ \\
\hline \multicolumn{5}{|l|}{ LY30, \% of clot lysis } \\
\hline Hyperfibrinolysis & $>\mathbf{3} \%$ & $1(12.5 \%)^{\mathrm{a}}$ & $>\mathbf{3} \%$ & $15(25.4 \%)$ \\
\hline Fibrinolysis Shutdown & $<0.9 \%$ & $5(62.5 \%)^{\mathrm{a}}$ & $<0.9 \%$ & $23(38.9 \%)$ \\
\hline
\end{tabular}

Bold values indicate statistical significance

Data presented as $n(\%)$

TEG thrombelastography, $A C T$ activated clotting time, $M A$ maximum amplitude, LY30\% of clot lysis at 30 min

${ }^{\mathrm{a}}=8$ out of the 19 patients had LY30 values available

Then, we combined as a total percent of patients with abnormal and normal TEG values to compare difference between the MT and the non-MT groups. When combining the total percentage of abnormal values from both centers, more
MT patients had a lower alpha-angle $(35.9 \%$ vs. $15.4 \%$, $p=0.023)$ and MA (43.6\% vs. $10.3 \%, p<0.001)$. Only one patient who received MT had an elevated alpha-angle. Furthermore, no patients who received MT had an elevated MA. 
There was no significant difference in percentage of abnormal ACT (at the r-TEG center only) or R-time (combined percentages) between those who received MT and those who did not.

Overall, 20 patients (48\%) at the k-TEG center did not have LY30 values available. After excluding those patients, we did not find a difference in the percentage of pediatric patients presenting with hyperfibrinolysis or fibrinolysis shutdown who received MT and those who did not. For the entire population who had LY30 values, 73\% (71/97) presented with a derangement in the fibrinolysis system $(26 \%$ with hyperfibrinolysis and $47 \%$ in fibrinolysis shutdown) (Table 3).

For the logistic regression analysis alpha-angle and MA were dichotomized as abnormal and normal based on their respective institutional normative values. After adjusting for age and GCS, on a univariable logistic regression model, a decreased MA significantly predicted MT [OR 3.68 (CI 1.29-10.52)] (Table 5).

\section{Discussion}

Pediatric trauma patients who undergo MT are more likely to have lower alpha-angles and MA values on TEG, as well as lower platelet counts and function. These findings support the use of TEG in pediatric trauma to help identify and promptly treat low fibrinogen and abnormal platelet function. These findings add to the growing body of the literature regarding the usefulness of TEG to appropriately guide blood product utilization in the management of severely injured pediatric patients $[6,8]$. Moreover, TEG provides real-time information on coagulation status, whereas, conventional coagulation studies can take up to one hour to result. In fact, to speed the flow of coagulation information during trauma resuscitations, both trauma centers utilize TEG software to view the TEG tracing in real-time in the trauma bay or the OR, as the sample is run in the lab.

Table 5 Logistic regression predicting massive transfusion

\begin{tabular}{llll}
\hline Predictors & \multicolumn{2}{l}{ Massive transfusion } \\
\cline { 2 - 4 } & Odds ratios & CI & $p$-values \\
\hline Age & 0.92 & $0.84-1.02$ & 0.104 \\
GCS & 0.94 & $0.87-1.01$ & 0.112 \\
$\alpha$-angle & 1.02 & $0.32-3.28$ & 0.970 \\
MA & 3.68 & $1.29-10.52$ & $\mathbf{0 . 0 1 5}$ \\
\hline
\end{tabular}

Bold values indicate statistical significance

CI confidence interval, GCS Glasgow coma score, MA maximum amplitude
Recently, our team developed the $A B C$ - $D$ score, to identify pediatric patients at risk for MT [2]. We showed that the addition of abnormal base deficit/lactate to the adult $\mathrm{ABC}$ score improved the sensitivity ( $>90 \%)$ in identifying children who will need MT [2]. Considering the results from our previous work combined with ability to view TEG in realtime offers potential for the development of a more accurate tool to identify and guide the resuscitation of pediatric patients who undergo MT.

A recent Cochrane Review showed that TEG-guided transfusion reduced overall mortality compared to transfusion guided by any method; however, the authors graded the evidence in pediatric patients as low-quality due to the small number of eligible studies [15]. Nevertheless, this review's results lend credence to other studies, which have shown that pediatric patients with traumatic coagulopathy are at a higher risk for mortality [16, 17]. A study by Ryan et al. evaluated coagulation abnormalities in pediatric trauma patients compared to age and gender-matched controls and found that pediatric trauma patients were more likely to have increased R-times, K-times, and alphaangles [18]. In contrast, our cohort included only patients who met highest level activation criteria. The patients in our study were more severely injured and found to have lower alpha-angles and MA values. Further work is needed to better understand the spectrum of coagulopathy (from hypo- to hyper- coagulopathy) in pediatric trauma patients [19].

The mechanisms underlying the coagulopathy seen in severely injured children with significant hemorrhage are poorly defined but include continual thrombin generation, which leads to platelet dysfunction [20-22]. In addition, the patients in our study who received MT had lower fibrinogen concentration on admission. Lower fibrinogen has been associated with increased transfusion requirements, ventilator days, and overall mortality [23]. Furthermore, the lower MA seen in MT patients demonstrates the inadequacy of platelet function to achieve hemostasis in these children with significant hemorrhage. Certainly, some of these patients may demonstrate platelet counts deemed acceptable during an acute trauma resuscitation, but the lower MA seen by TEG highlights the important of adequate platelet resuscitation for dysfunction (and not simply for platelet count). Others have proposed a concept of platelet exhaustion in trauma as an etiology of platelet dysfunction (despite adequate number) [23, 24]. Our work agrees with this concept of platelet exhaustion. It adds to a growing body of evidence of TEG's importance in the management of pediatric trauma patients, specifically, the importance of the MA portion of the tracing. Leeper et al. identified an $\mathrm{MA}<55$ as a trigger for transfusion in pediatric trauma patients [17]. Vogel et al. found MA was independently associated with increased mortality in pediatric 
trauma patients [8]. Our current data shows MA indicates a patient at higher risk for undergoing MT. These studies collectively support the ACS recommendation to use TEG in pediatric trauma patients [12]. Unfortunately, only one in five trauma centers report routinely incorporating pointof-care TEG's into their MTP policies [13].

Potential limitations to our study include its retrospective nature and the small number of patients. There is the potential for bias because we evaluated TEG in a high-risk patient population, which consists of the highest-level pediatric trauma activations. Therefore, it is yet unclear if these results are generalizable to all pediatric trauma patients. We did not specifically examine the optimal thresholds to guide hemostatic resuscitation in pediatric trauma patients using TEG. Furthermore, each institution uses different TEG devices with slightly different parameters. It is impossible to compare the impact of the different technologies within the constructs of a small retrospective study.

We conclude that severely injured pediatric patients have unique TEG abnormalities upon admission. Specifically, those who undergo MT are more likely to have lower alpha-angles and MA values, which suggest they may benefit from earlier cryoprecipitate or platelet administration. The need for real-time and ongoing monitoring of these patients' coagulation status using k-TEG or r-TEG appears to be critical in reducing the morbidity and mortality of pediatric trauma patients undergoing MT and warrants prospective validation.

Author contribution Study conception and design: Ryan Phillips, Hunter Moore, Denis Bensard, Niti Shahi, John Kim, Steven Moulton. Acquisition of data: Ryan Phillips, Niti Shahi, Gabrielle Shirek, Steven Moulton. Analysis and interpretation of data: Ryan Phillips, Hunter Moore, Denis Bensard, Niti Shahi, Maxene Meier, John Kim, Steven Moulton. Drafting of the manuscript: Ryan Phillips, Hunter Moore, Niti Shahi, Marina L. Reppucci, Gabrielle Shirek. Critical revision of manuscript: Ryan Phillips, Hunter Moore, Denis Bensard, Niti Shahi, Marina L. Reppucci, Gabrielle Shirek, Maxene Meier, Shannon Acker, John Kim, Steven Moulton

\section{Declarations}

Conflicts of interest None of the authors has a conflict of interest to disclose.

Ethical approval All of the procedures performed in studies involving human participants were in accordance with the ethical standards of the institutional and/or national research committee and with the 1964 Helsinki Declaration and its later amendments or comparable ethical standards.

Informed consent The study was evaluated and approved by the Colorado Multiple Institution Review Board (COMIRB), and a waiver of informed consent as obtained due to the retrospective nature of the study.

\section{References}

1. Nystrup KB, Stensballe J, Bøttger M, Johansson PI, Ostrowski SR (2015) Transfusion therapy in paediatric trauma patients: a review of the literature. Scand J Trauma Resusc Emerg Med 23(1):21

2. Phillips R, Acker SN, Shahi N, Meier M, Leopold D, Recicar J et al (2020) The ABC-D score improves the sensitivity in predicting need for massive transfusion in pediatric trauma patients. J Pediatr Surg 55(2):331-334

3. Rosenfeld EH, Lau P, Cunningham ME, Zhang W, Russell RT, Naik-Mathuria B et al (2019) Defining massive transfusion in civilian pediatric trauma with traumatic brain injury. J Surg Res 1(236):44-50

4. Dehmer JJ, Adamson WT (2010) Massive transfusion and blood product use in the pediatric trauma patient. Semin Pediatr Surg 19(4):286-291

5. Noland DK, Apelt N, Greenwell C, Tweed J, Notrica DM, Garcia NM et al (2019) Massive transfusion in pediatric trauma: an ATOMAC perspective. J Pediatr Surg 54(2):345-349

6. Leeper CM, Kutcher M, Nasr I, McKenna C, Billiar T, Neal M et al (2016) Acute traumatic coagulopathy in a critically injured pediatric population: definition, trend over time, and outcomes. J Trauma Acute Care Surg 81(1):34-41

7. Liras IN, Caplan HW, Stensballe J, Wade CE, Cox CS, Cotton BA (2017) Prevalence and impact of admission acute traumatic coagulopathy on treatment intensity, resource use, and mortality: an evaluation of 956 severely injured children and adolescents. J Am Coll Surg 224(4):625-632

8. Vogel AM, Radwan ZA, Cox CS Jr, Cotton BA (2013) Admission rapid thrombelastography delivers real-time "actionable" data in pediatric trauma. J Pediatr Surg 48(6):1371-1376

9. Holcomb JB, Minei KM, Scerbo ML, Radwan ZA, Wade CE, Kozar RA et al (2012) Admission rapid thrombelastography can replace conventional coagulation tests in the emergency department: experience with 1974 consecutive trauma patients. Ann Surg 256(3):476-486

10. Gonzalez E, Moore EE, Moore HB, Chapman MP, Chin TL, Ghasabyan A et al (2016) Goal-directed hemostatic resuscitation of trauma-induced coagulopathy: a pragmatic randomized clinical trial comparing a viscoelastic assay to conventional coagulation assays. Ann Surg 263(6):1051

11. Lammers DT, Marenco CW, Morte KR, Bingham JR, Martin MJ, Eckert MJ (2020) Viscoelastic testing in combat resuscitation: is it time for a new standard? J Trauma Acute Care Surg. https://doi.org/10.1097/TA.0000000000002634

12. Camazine MN, Hemmila MR, Leonard JC, Jacobs RA, Horst JA, Kozar RA et al (2015) Massive transfusion policies at trauma centers participating in the American College of Surgeons Trauma Quality Improvement Program. J Trauma Acute Care Surg 78(6):S48-53

13. Etchill E, Sperry J, Zuckerbraun B, Alarcon L, Brown J, Schuster $\mathrm{K}$ et al (2016) The confusion continues: results from an American Association for the Surgery of Trauma survey on massive transfusion practices among United States trauma centers. Transfusion 56(10):2478-2486

14. Salooja N, Perry DJ (2001) Thrombelastography. Blood Coagul Fibrinolysis 12(5):327-337

15. Wikkels $\varnothing$ A, Wetterslev J, Møller AM, Afshari A (2016) Thromboelastography (TEG) or thromboelastometry (ROTEM) to monitor haemostatic treatment versus usual care in adults or children with bleeding. Cochrane Database Syst Rev. https:// doi.org/10.1002/14651858.CD007871.pub3

16. Reed CR, Williamson H, Vatsaas C, Kamyszek R, Leraas HJ, Ray $\mathrm{C}$ et al (2019) Higher mortality in pediatric and adult 
trauma patients with traumatic coagulopathy, using age-adjusted diagnostic criteria. Surgery 165(6):1108-1115

17. Leeper CM, Neal MD, McKenna C, Billiar T, Gaines BA (2018) Principal component analysis of coagulation assays in severely injured children. Surgery 163(4):827-831

18. Ryan ML, Van Haren RM, Thorson CM, Andrews DM, Perez EA, Neville HL et al (2014) Trauma induced hypercoagulablity in pediatric patients. J Pediatr Surg 49(8):1295-1299

19. Gando S, Hayakawa M (2016) Pathophysiology of trauma-induced coagulopathy and management of critical bleeding requiring massive transfusion. Semin Thromb Hemos 42(2):155-165

20. Moore HB, Walsh M, Kwaan H, Medcalf RL (2020) The complexity of trauma-induced coagulopathy. Semin Thromb Hemos 46(02):114-115

21. Wohlauer MV, Moore EE, Thomas S, Sauaia A, Evans E, Harr J et al (2012) Early platelet dysfunction: an unrecognized role in the acute coagulopathy of trauma. J Am Coll Surg 214(5):739-746
22. Curry N, Foley C, Wong H, Mora A, Curnow E, Zarankaite A et al (2018) Early fibrinogen concentrate therapy for major haemorrhage in trauma (E-FIT 1): results from a UK multi-centre, randomised, double blind, placebo-controlled pilot trial. Crit Care 22(1): 164

23. Cardenas JC, Zhang X, Fox EE, Cotton BA, Hess JR, Schreiber MA et al (2018) Platelet transfusions improve hemostasis and survival in a substudy of the prospective, randomized PROPPR trial. Blood Adv 2(14):1696-1704

24. Martin GE, Pugh AM, Moran R, Veile R, Friend LA, Pritts TA et al (2019) Microvesicles generated following traumatic brain injury induce platelet dysfunction via adenosine diphosphate receptor. J Trauma Acute Care Surg 86(4):592-600

Publisher's Note Springer Nature remains neutral with regard to jurisdictional claims in published maps and institutional affiliations. 